\title{
Intronic MiRNA MiR-3666 modulates its host gene FOXP2 functions in neurodevelopment and may contribute to pathogenesis of neurological disorders schizophrenia and autism
}

\begin{abstract}
MicroRNAs (miRNAs) are approximately 22-nucleotide-long, non-coding RNAs that bind to complementary mRNAs with inhibitory effect. An intronic miRNA is embedded in a particular gene called its host gene. Our study focuses on the Homo sapiens intronic miRNA-host gene pair, hsa-miR-3666 and FOXP2. Previous report of co-expression of miR-3666 and FOXP2 indicates possible regulation of FOXP2 functions by miR-3666. However, direct correlation has not been shown yet. Therefore, we took a computational approach to determine if and how such modulation occurs. ChIP-seq identified FOXP2 targets and putative miR-3666 targets showed a significant overlap of 574 common target genes. Functional enrichment analysis of common targets revealed over-representation of KEGG pathways and Gene Ontology modules associated with neurodevelopment. These modules, along with further literature mining and protein-protein interaction analysis of FOXP2 and miR3666 identified several specific genes associated with neurodevelopment and finally integration of transcriptomic expressions data lead to the selection of four models depicting the mechanisms by which miR-3666 can modulate FOXP2 functions. Model 1 illustrates that during neurodevelopment, miR-3666 can directly modulate the functions of FOXP2 through regulation of common targets, such as IGF1 and EFNB2, whereas model 2 shows miR-3666 can also indirectly modulate FOXP 2 functions by considering targets that are not common for the intronic miRNA-host gene pair, for example $\mathrm{CDH} 2$ and $\mathrm{LMO} 4$. This direct and indirect regulation is necessary for precise spatial and temporal expression of genes during neurodevelopment. Models 3 and 4 exhibit mechanisms in which the interactions of miR-3666 and FOXP2 with target genes contribute to the pathogenesis of schizophrenia and autism respectively.
\end{abstract}

Keywords: FOXP2, miR-3666, target genes, interaction, co-regulation, neurodevelopment, schizophrenia, autism, autism spectrum disorder
Volume 2 Issue I - 2017

\author{
Mohd Mostafa S,' Wahid Murad M,' Eusra \\ Mohammad, ${ }^{1,2}$ Islam ABMMK' \\ 'Department of Genetic Engineering and Biotechnology, \\ University of Dhaka, Bangladesh \\ 2Department of Mathematics and Natural Sciences, BRAC \\ University, Bangladesh
}

Correspondence: Abul Bashar Mir Md. Khademul Islam, Associate Professor, University of Dhaka, Department of Genetic Engineering \& Biotechnology, University of Dhaka, Dhaka-1000, Bangladesh, Tel +880-2-9661900, extn 7825, Email khademul@du.ac.bd

Received: November 21, 2016 | Published: February 07, 2017
Abbreviations: ASD, autism spectrum disorder; DN, differentiated neuron; FC, fold change; GEO, gene expression omnibus; $\mathrm{GOBP}$, gene ontology biological process; GOCC, gene ontology cellular component; GOMF, gene ontology molecular function; HESC, human embryonic stem cell; IPC, intermediate progenitor cell; miRNA, microRNA; NE, neural ectoderm; NPC, neural progenitor cell; NSC, neural stem cell; NT2, NTera2; PHS, pitt-hopkins syndrome; $\mathrm{TF}$, transcription factor

\section{Introduction}

Mature microRNAs (miRNAs) are 18-22 nucleotides long and are known to repress translation by binding to the complementary 3'-UTRs of their target mRNAs. This binding results in inhibition of translation initiation or post-initiation translational block. Each miRNA can recognize multiple target mRNAs that may be related to one or more biological processes; hence miRNA can have diverse effects. ${ }^{1}$ Our study involves a type of miRNA called intronic miRNA that is embedded in the introns of its "host gene". The definition of intronic miRNA depends on two parts: first, sharing the same promoter with their encoded genes and second, being spliced out of the transcript of their encoded genes and further processed into mature miRNAs. ${ }^{2}$
These intronic miRNAs may support or counteract the functions of its host gene. ${ }^{3}$ Forkhead genes are a subgroup belonging to the helixturn-helix class of proteins. ${ }^{4}$ FOXP2 (Forkhead box P2) protein contains a FOX DNA-binding domain and a large polyglutamine tract and is evolutionarily conserved, binding directly to 300 to 400 gene promoters in the human genome and hence can regulate a variety of genes. ${ }^{5}$ FOXP2 can form homodimers and heterodimers with FOXP1 and FOXP4; this dimerization is a requirement for DNAbinding. ${ }^{6}$ Heterodimers of FOXP2 with FOXP1 may have different transcriptional outcomes than their homodimers. Hence, situations may arise where low levels of $F O X P 2$ could repress transcription by heterodimerization with FOXP1, but as FOXP2 increases in amount, competition between FOXP2 homodimers and endogenous FOXP1 can lead to transcriptional activation. ${ }^{7}$ FOXP2 is said to have dual functionality, either repressing or activating gene expression. ${ }^{8}$ It generally works as a repressor, however, its overexpression has been shown to increase expression of some genes such as TAGLN and CER1, suggesting a role in transcriptional activation. ${ }^{7}$ FOXP2 hosts the intronic miRNA, miR-3666. Though not much work has been done on miR-3666, its targets have been predicted and deposited in various databases. A few recent experiments have shown the repression 
activity of miR-3666 on targets such as MET and ZEB1 in thyroid carcinoma and cervical carcinoma cells, respectively. ${ }^{9,10}$

Previous experiments ${ }^{11,12}$ have demonstrated how intronic miRNA can modulate host gene functions. Since little research has been done with miR-3666, its potential functions with respect to modulation of host gene activities are largely unknown. Furthermore, the co-expression of FOXP2 and miR-3666 has not only been computationally predicted; it has also been confirmed in vitro. ${ }^{1} \mathrm{We}$ therefore sought to study if miR-3666 can play an antagonistic or synergistic role in regulation of FOXP2 functions and if it can, the mechanisms by which it does so. This regulatory function of intronic miRNA has critical implications in the designing of therapeutics or its role as biomarkers. miRNAs have been shown to be effective as therapeutics due to several reasons such as its small, conserved sequence; its high binding specificity and affinity; and overall desirable pharmacokinetic properties. ${ }^{13}$ Circulating miRNAs have also been considered good candidates as biomarkers. ${ }^{14}$ In this study, we took a "data-driven and knowledge-based approach" to find functional relations between intronic miRNA miR-3666 and its host gene, FOXP2. We hypothesized that the presence of common target genes for miR-3666 and FOXP2 mean that they are involved in regulation of a common pathway or biological function. Moreover, miR-3666 was also expected to have a synergistic or antagonistic effect on host gene function. Functional enrichment analysis of KEGG pathways and Gene Ontology (GO) on common targets from ChIP-seq experiments highlighted pathways, biological processes and sets of genes that are enriched for both miRNA and host gene.

\section{Methods}

\section{Identification of hsa-mir-3666 (miR-3666) and FOXP2 targets}

Several miRNA target databases were searched for putative targets of miR-3666 which includes TargetScan (www.targetscan.org) (Release 6.2: June 2012), ${ }^{15}$ TarBase (diana.imis.athena-innovation.gr/ DianaTools), ${ }^{16}$ PicTar (http://pictar.mdc-berlin.de) ${ }^{17}$ and miRecords (c1.accurascience.com/miRecords/) ${ }^{18}$ (Figure 1). ChIP-seq experiment datasets of FOXP2 were downloaded from Encyclopedia of DNA Elements (ENCODE) ChIP-seq Experiment Matrix (human genome version hg19) dataset UCSC 2003-2012. ${ }^{19}$ The closestBed feature of BEDTools ${ }^{20}$ was used to identify nearest Ensembl (version 70$)^{21}$ transcript from the ChIP-seq peak as the target gene.

\section{Overlap analysis}

Overlap analysis of the targets of FOXP2 and that of miR-3666 was carried out using the tool Venny (version 2.1.0). ${ }^{22}$ Significance of overlap analysis was based on Chi-square test.

\section{Literature mining and expression data analysis}

Extensive text-mining related to both $F O X P 2$ and miR-3666 revealed their respective functions at the molecular level. Knowledge about the pathways and processes they are directly or indirectly involved; in their spatial and temporal expression patterns; and the disorders that may result due to perturbations in their function or expression, was used to create the basis upon which our models were developed. The expression profile of the selected genes across various stages of neurodevelopment were obtained from GEO series GSE28633. ${ }^{23}$ The data were $\log _{2}$-transformed and the expression values of multiple probes of the same gene were averaged, these values were then visualized as a heatmap using Matrix2png. ${ }^{24}$ For differential expression, the samples were placed into test and control groups and GEO2R analysis was carried out with default parameters. $\log _{2} \mathrm{FC}$ (fold change) cut off was set to -0.5 and +0.5 for significant differential expression. OncoDrive analysis of Gitools ${ }^{25}$ was used for the detection of "driver genes". The results of the driver gene analysis were visualized as a heatmap in Gitools using P value scale with corrected right $\mathrm{p}$-value less than 0.05 . To obtain expression profile of miR-3666, Gene Expression Omnibus (GEO) ${ }^{26,27}$ series "GSE158881" was subjected to GEO2R analysis. The samples in GSE15888 were placed into defined groups and the test was run using default parameters.

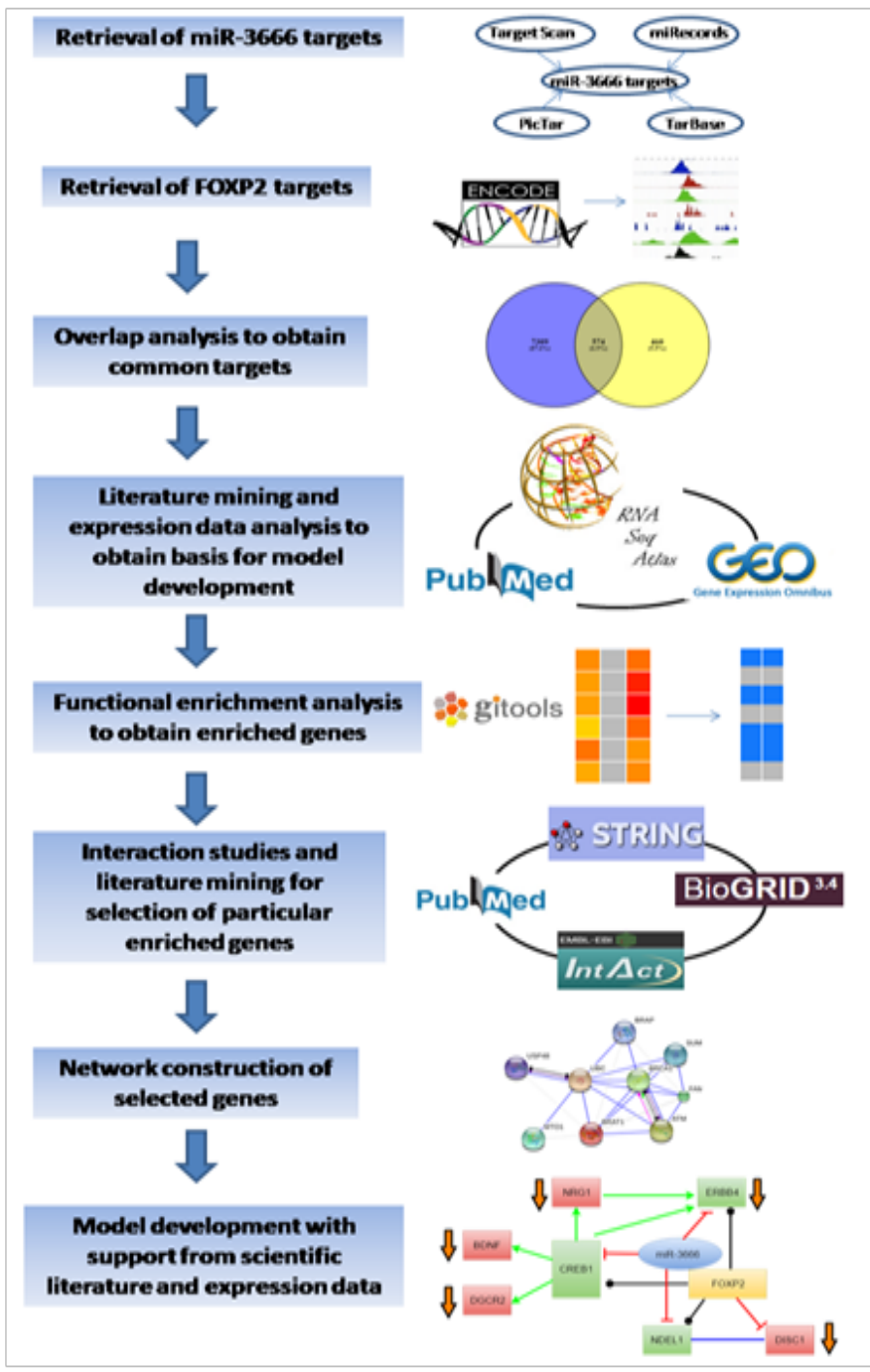

Figure I Overview of process of developing models to depict the role of miR-3666 in modulation of host gene functions.

\section{Functional enrichment analysis}

Functional annotation of target genes is based on Gene Ontology $(\mathrm{GO})^{28}$ as extracted from EnsEMBL ${ }^{29}$ and KEGG pathway database. ${ }^{30}$ Accordingly, all genes are classified into the ontology categories biological process (GOBP) and pathways when possible. We have taken only the GO/pathway categories that have at least 10 genes annotated. We used Gitools for enrichment analysis and heatmap generation. ${ }^{25}$ Resulting p-values were adjusted for multiple testing 
(p-value less than 0.01) using the Benjamin and Hochberg's method of False Discovery Rate (FDR). ${ }^{31,32}$ The candidate gene list for schizophrenia was collected from Schizophrenia Gene Resource ${ }^{33}$ whereas candidate genes for autism were collected from Autism $\mathrm{KB}^{34}$ and SFARI gene databases. ${ }^{35}$ Considering the candidate genes, we carried out enrichment analysis to find whether the disorders are significantly enriched when common target genes are considered.

\section{Selection of genes and development of models}

In order to construct a model to demonstrate how the intronic miRNA miR-3666 affects the function of its host gene, we selected a particular set of genes. These genes were selected based on their significance in neurodevelopment and potential in being directly or indirectly affected by FOXP2 and miR-3666. Protein-protein interaction studies were done using STRING (version 10.0), ${ }^{36,37}$ BioGRID (version 3.4) ) $^{38}$ and IntAct ${ }^{39}$ databases. STRING database was used to build a protein-protein interaction network model keeping all parameters in default.

\section{Results and discussion}

\section{Host and intronic miRNA targets overlap significantly}

First we sought to find the target genes of miR-3666. Since miR-3666 has only recently been added to miRNA databases, experimentally validated targets are not available yet. The targets of miR-3666 downloaded from major miRNA target databases-Tarbase, TargetScan, PicTar, miRecords were combined to form a unique union set of 1028 target genes. This approach allowed a comprehensive method in determination of putative targets of miR-3666 and therefore an increased confidence in the results. Similarly, by analyzing FOXP2 ChIP-seq data in SK-N-MC cell line we identify total 7883 FOXP2 targets. In order to study the role of miR-3666 in modulating its host gene functions, we analyzed the target genes that are affected by the action of both host gene and intronic miRNA. Overlap analysis in a venn-diagram showed that miR-3666 and FOXP2 have 574 common targets (Figure 2A). Chi-square test shows that the overlap is significant ( $\mathrm{p}$-value $<10^{-16}$ and Chi-square value 1817.16) and much higher than expected value (percentage deviation is $+351.3 \%$ )

\section{FOXP2 and miR-3666 perform common role in neuro- development}

FOXP2 has widespread expression in humans (Supplementary Figure S1). It is expressed at high levels in the developing brain, with lower expression in various parts of the human brain. Besides the brain, it is expresses in the lungs and gut as well. ${ }^{40,41}$ Although FOXP2 has extensive expression in the developing brain, a quite low expression in the adult brain $^{42}$ suggests that the expression of FOXP2 is developmentally regulated. Mutational analysis in several studies $^{43-45}$ have demonstrated the association of non-functional FOXP2 with motor dysfunction, cerebellar abnormalities and early postnatal lethality. In an experiment that studied miRNA expression during the process of neural differentiation using an RA (retinoic acid)-induced embryonal carcinoma NTera2/D1 (NT2) cell line, miR3666 was observed to continue being expressed in fully differentiated NT2-derived post-mitotic neurons and/or NT2-derived astrocytes. The differential expression of miR-3666 during the experiment indicated its role in regulation of neurodevelopment, particularly the peak in miR-3666 expression between 6 and 14 days of treatment implies a biological role in cell-fate determination ${ }^{1}$ (Supplementary Figure S2).
Above results and information indicates that miR-3666 and FOXP2 are involved in neurodevelopment so that our analysis focused on FOXP2 and miR-3666 co-regulation of neurodevelopmental processes.

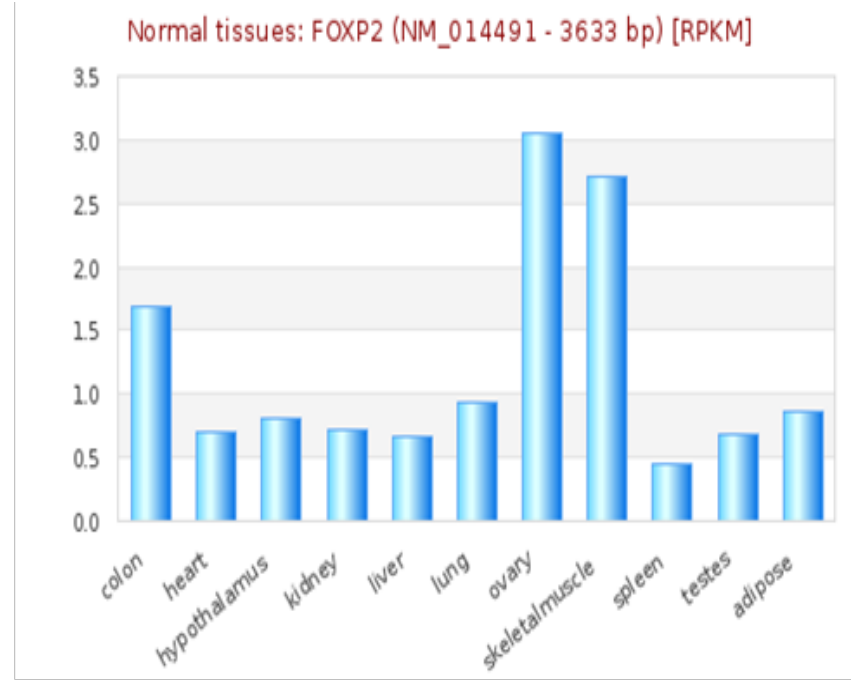

Supplementary figure SI RNA -seq atlas displaying relative levels of FOXP2 expression across various organs. The height of blue bars represents the expression levels of FOXP2 [source: RNA-Seq Atlas].

\section{Over-represented Gene Ontologies and KEGG pa- thways of common targets are related to neurodeve- lopment, which are not modulated by miR-3666 lone targets}

Enrichment analysis allows us to understand and quantitatively measure whether there are statistically significant changes in a set of biological annotations. We sought to determine the host gene functions that are modulated by intronic miRNA, miR-3666. Hence we have done enrichment analysis using Gitools ${ }^{25}$ to obtain significantly overrepresented Gene Ontology Biological Process (GOBP) terms (Figure $2 \mathrm{~B}$ ) and KEGG pathways (Figure $2 \mathrm{C}$ ) and on the common and unique targets of FOXP2 and miR-3666.Although functional enrichment analysis showed enrichment for a diverse set of processes (Figure 2B $\& 2 \mathrm{C}$ ) at a very low p-value of 0.01 , there is a clear predominance of functions such as "neuron differentiation"; neurological disorders; and signaling pathways involved in neurodevelopment, neurogenesis or gliogenesis in commonly regulated targets of FOXP2 and miR-3666. All of the enriched KEGG pathways and GO Biological Processes were then analyzed for related enriched genes. By combining all the enriched genes obtained from both KEGG and GOBP modules, we hence obtained 249 common target genes that were significantly enriched for all pathways and processes related to neural development (Supplementary Table S3).

Language disorder and cognitive deficiency is commonly considered as one of the principal symptoms in several disorders, such as schizophrenia and autism. Hence, FOXP2 is likely to be a good candidate gene since its mutation has been reported to be associated with speech and language deficits. ${ }^{46-48}$ FOXP2 has also been reported to be associated with schizophrenia ${ }^{49-52}$ and autism. ${ }^{53-56}$ FOXP2 may therefore directly cause the disease or affect its downstream targets that lead to the development of such disorders. We see that, when considering candidate genes of the disorders and common targets of FOXP2 and miR-3666, both schizophrenia and autism are significantly enriched (Figure 2D). 


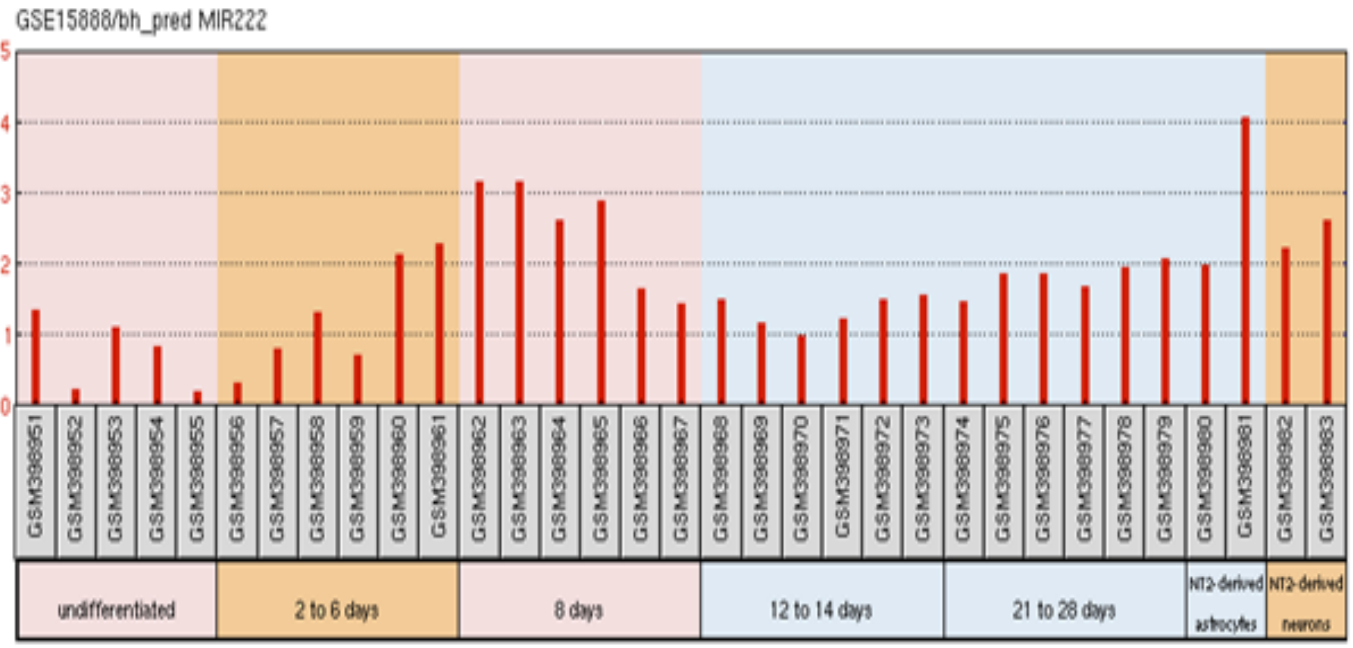

- expression value

Supplementary figure S2 miR-3666 expression profile in NT2/DI.

The profile diagram is titled by the GEO accession number (GSEI5888) and the probe ID (bh_pred MIR222). The groups shown are

I. Undifferentiated cells; cells harvested

II. 2, 4 and 6 days after retinoic acid (RA) treatment

III. 8 days after RA treatment

IV. cells harvested 12 and I4 days after RA treatment

V. $2 \mathrm{I}$ and 28 days after RA treatment

VI. NT2-derived astrocytes and

VII. NT2-derived neurons. Each group contains particular samples as designated by their GSM IDs.

VIII. The red bars represent the expression measurement extracted from the median-centered, log 2 signal intensity values column of the samples.

Four models developed show mechanisms by which miR-3666 might modulate FOXP2 functions in neurodevelopment and pathogenesis of neurological disorders

A thorough study of previous FOXP2 and neurodevelopmentrelated research revealed several key genes for neurodevelopment. Previous experiments that identified FOXP2 targets using methods such as ChIP-chip and ChIP-quantitative $\mathrm{PCR}^{7,8,57,58}$ show FOXP2 targets are enriched in functions related to synaptic plasticity, neurotransmission and axon guidance. Some of these targets have also reported to be differentially expressed in humans and have been associated with cognitive disorders such as autism. ${ }^{7}$ Hence, we selected common target genes that may have these exact or related functions. Additionally, proliferation and differentiation in neurogenesis implies that cell cycle re-entry and exit is also involved. Hence, we also selected common target genes that are crucial in the cell cycle, for example, cyclin D3 (CCND3). ${ }^{59}$ We also studied FOXP2 interactions using STRING, ${ }^{36,37}$ (Figure 3A), BioGRID ${ }^{38}$ and IntAct. ${ }^{64}$ For a more extensive study, we referred scientific literature ${ }^{60-69}$ and took into consideration some genes that were not common targets but play important roles in the nervous system or in pathogenesis of neurological disorders (for example, CNTNAP2 and $B D N F$ ) and showed potential in being indirectly co-regulated by FOXP2 and miR3666 through common target genes. Therefore, a total of 30 genes were selected for developing our models (Supplementary Table S4). STRING $^{36,37}$ analysis of the proteins of selected genes (Figure 3B) highlights important interactions, for example, the binding of DISC1 (FOXP2 target) with NDEL1 (common target gene). Additionally, it shows MET and ERBB4 proteins may act as hubs. Since STRING did not display interactions for all proteins (for example, SOX21), we carried out interactions studies using other databases, namely
BioGRID $^{38}$ and IntAct ${ }^{39}$ for a more thorough analysis. We compiled a list of candidate genes for schizophrenia and autism from different databases and subjected them to overlap analysis with our list of selected 30 genes (Figure 4) (Supplementary Table S5). Models were developed based on the selected genes that have been linked to the particular disorder.

With this list of genes we have developed 4 models. Models 1 and 2 depict the joint regulation of miR-3666 and FOXP2 in neurodevelopmental processes. Model 1 (Figure 5A) shows interactions of miR-3666 and FOXP2 with mainly the common target genes and genes that have regulatory effects on FOXP2. Model 2 (Figure 5B) involves many target genes that are either unique to miR-3666 or FOXP2 but not both. However, model 2 shows how these genes may be indirectly regulated by both the host gene and the intronic miRNA. In models 3 and 4, we propose how FOXP 2 and miR3666 can jointly regulate the expression of these candidate genes and influence the pathogenesis of schizophrenia (Figure 5C) and autism and ASD (Autism Spectrum Disorder) (Figure 5D), respectively.

\section{miR-3666 directly or indirectly regulates FOXP2 func- tions in neuronal differentiation}

As model 1 (Figure 5A) shows, miR-3666 can regulate neuron differentiation by directly regulating the ChIP-Seq targets of FOXP2NEUROD1, IGF1, CCND3 and SOX4. FOXP2 may control neuronal differentiation by interacting with NEUROD1; miR-3666 regulates this function by directly inhibiting NEUROD1 itself or its activators, NEUROG1 and NEUROG2. Additionally, inhibition of the FOXP2 targets CCND3 and IGF1 by miR-3666 may promote cell cycle exit and neural differentiation. The co-regulation of SOX4 by miR-3666 and FOXP2is important for the proper transition from radial precursor to intermediate progenitor cells (IPCs). ${ }^{70}$ As model 2 (Figure 5B) 
shows, ASCL1 may be indirectly regulated by both FOXP2 and miR-3666 via common targets TCF4 ${ }^{71}$ and HES1. ${ }^{72,73}$ Though the ChIP-seq data we used did not list ASCL1 as an experimentally determined target, literature review has revealed that FOXP2 strongly represses ASCL1. ${ }^{74}$ Additionally, the joint regulation of the common target SOX21 by miR-3666 and FOXP2 maintains the balance of SOX2 and SOX21 activities that, in turn, is required for the balance of progenitor cell maintenance and the progression to postmitotic neural development. ${ }^{75,76}$ Furthermore, downregulation of

Supplementary Table S3 List of FOXP2 and miR-3666 target genes that are enriched for neural development related terms in GOBP and KEGG pathways
$\mathrm{CDH} 2$ is necessary for neuronal differentiation to occur. ${ }^{66}$ FOXP2 directly represses $\mathrm{CDH} 2$, leading to detachment of differentiating neurons from epithelial sheet; ${ }^{77}$ we presume miR-3666 may suppress $\mathrm{CDH} 2$ indirectly via suppression of Protein Tyrosine Phosphatase, Receptor Type, J (PTPRJ). According to IntAct database, PTPRJ dephosphorylates CDH2. PTPRJ may act similarly to PTP1B (another phosphatase) by maintaining cells in an adhesion-competent state by dephosphorylating $\beta$-catenin. . $^{7,79}$

\begin{tabular}{|c|c|c|c|c|c|c|c|}
\hline AAKI & CDC73 & ERBB4 & KIT & NDELI & PRKD3 & sox 4 & TRIB I \\
\hline ABCC4 & CEBPE & EREG & LDLRAD3 & NEDD4L & PRNP & SOX5 & TRIM2 \\
\hline ACVRI & CEPI20 & FBXWII & LDLR & NEURODI & PSD3 & SPI & TRPC3 \\
\hline ADCYI & CHRM2 & FERMT2 & LIN28A & NFIB & PTPNII & SPATA2 & $\mathrm{TSCl}$ \\
\hline AGFGI & CHSTII & FMRI & LMLN & NPNT & PTPRD & SPEN & TTPA \\
\hline AHR & CLTC & FNBPI & LRPI2 & NPTN & QKI & SPHK2 & TXNIP \\
\hline AK4 & COLI9AI & FOXPI & LRPIB & $\mathrm{NR} 3 \mathrm{Cl}$ & RAB5B & SPOCKI & UHMKI \\
\hline APCDDI & COL6A3 & FRMD6 & LRP6 & NRPI & RACGAPI & SPREDI & ULK2 \\
\hline ARHGAP24 & COX7A2L & FRZB & LRP8 & NRP2 & RALBPI & SRGAP3 & UNCI3A \\
\hline ARHGEFI 2 & CPEB2 & FZD3 & LRRK2 & NRSNI & RAPGEF4 & STAT3 & VANGLI \\
\hline ARID5B & CPEB4 & GABI & MAFB & NUSI & RNF4I & STAT6 & VAPA \\
\hline ARL4A & CREBI & GCLC & MAGII & OTX2 & $\mathrm{ROBO} 2$ & STIM2 & VAV2 \\
\hline ARX & CREB5 & GDA & MAML3 & PDE4D & ROCK2 & STK4 & WASL \\
\hline ATP2A2 & CUL3 & GJAI & MAP3KI3 & PDE5A & RPS6KA2 & SULFI & WHSCILI \\
\hline ATP6VIB2 & CXCLI2 & GNAI 2 & MAP4 & PDE7B & RRAGD & SYT2 & WNTIOA \\
\hline ATXN I & DCBLD2 & GRIK2 & MAP7 & PDE8A & RTNI & TACCI & WNT2B \\
\hline BAG5 & DENNDIA & HBPI & MAPKIO & PI4KA & RUNXITI & TAF4B & XYLTI \\
\hline BCLIIA & DICERI & HECA & MAPKI & PIK3C2A & RXFP2 & TAF4 & YESI \\
\hline BHLHE4I & $\mathrm{DLCl}$ & HEG I & MBD2 & PIK3IPI & SIPRI & TANCI & ZBTB7B \\
\hline BMPRIB & DLG5 & HESI & MBNLI & PLEKHG5 & SIPR2 & TAOKI & ZEBI \\
\hline BMPR2 & DNM2 & HOMERI & MDFIC & PLEKHG5 & SBF2 & TBLIXR I & ZEB2 \\
\hline BPTF & DPYSL2 & HSPA8 & MDGA2 & PLLP & SETD7 & TBPLI & ZFAND5 \\
\hline BTG I & EDA & IGFI & MEOX2 & PMEPAI & $\mathrm{SH} 2 \mathrm{~B} 3$ & TCF4 & ZFPM2 \\
\hline CABLESI & EFNB2 & IGFBP5 & MET & POU3F2 & SHANK2 & TENMI & $\mathrm{ZIC5}$ \\
\hline CALB I & EGR3 & INHBB & MLL & POU4FI & $\mathrm{SHC} 3$ & TFDP2 & ZNF3 \\
\hline CALMI & $\mathrm{EIF} 2 \mathrm{Cl}$ & ITGA4 & MLTK & PPARGCIA & SIKI & TGFB2 & ZNRF3 \\
\hline CALM2 & EMX2 & ITGB8 & MYB & PPFIA2 & SIPAIL2 & TGFBR2 & \\
\hline CANX & ENAH & ITPKB & MYOIO & PPPIR9A & SMAD2 & THSD7A & \\
\hline CAPRIN2 & EPB4ILI & JARID2 & MYTIL & PRICKLE2 & SMAD5 & TIMP2 & \\
\hline CAV2 & EPDRI & KCNN3 & NAVI & PRKAAI & SNAP25 & TNRC6B & \\
\hline CCDC88A & EPHA7 & KIAAI 462 & NCOAI & PRKABI & SNX2 & TNRC6C & \\
\hline CCND3 & EPSI5 & KIFI3A & NCOA3 & PRKACB & SOX2I & TP63 & \\
\hline
\end{tabular}



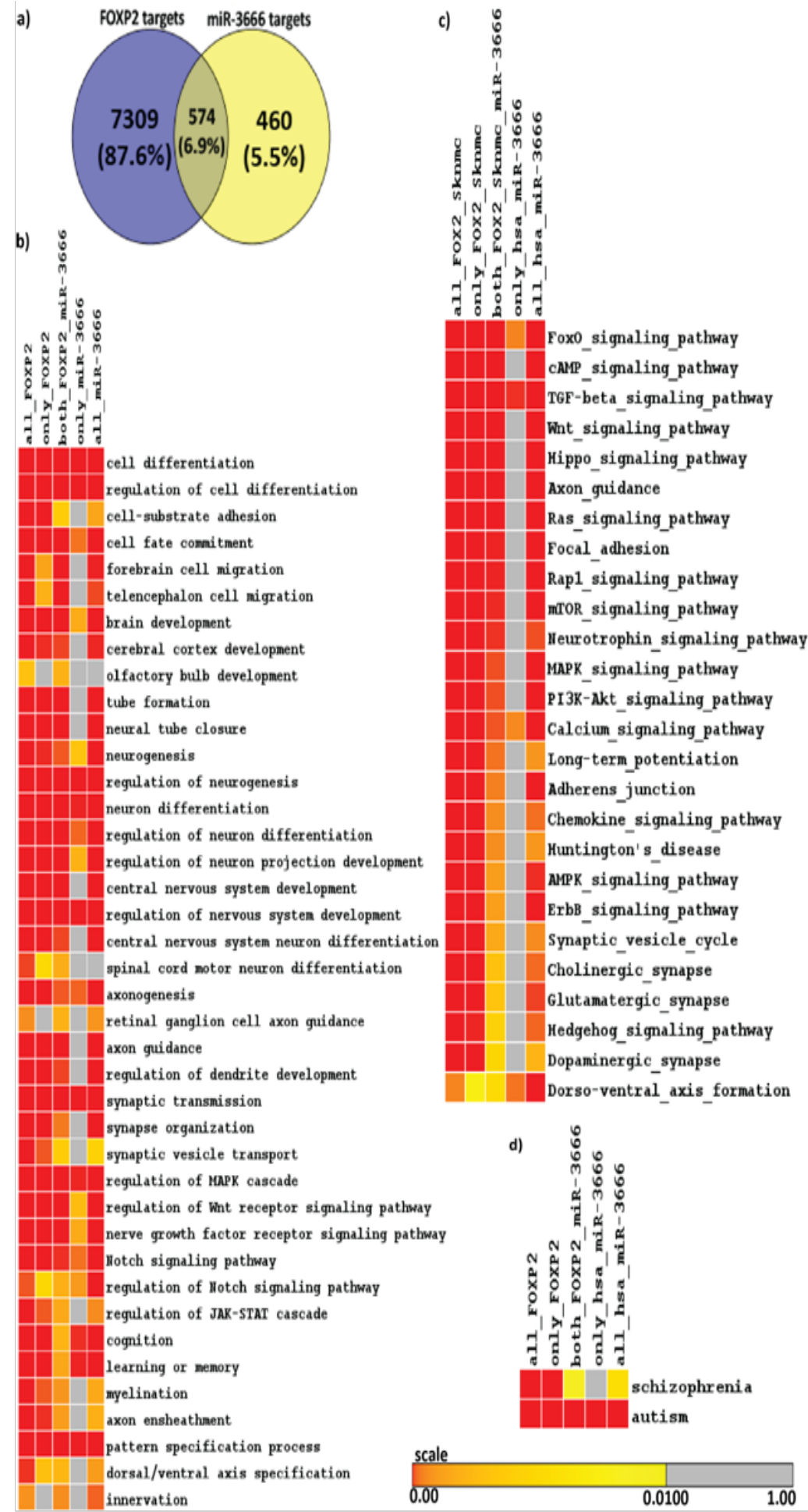

Figure 2 Identification of FOXP2 and miR-3666 common targets and functional overrepresentation analysis of targets.

A. venn representation of common and unique targets genes of host genes and intronic miRNA. The left circle represents FOXP2 target genes, where the number in the blue circle corresponds to genes exclusive for FOXP2. The right circle represents miR-3666 target genes, where the number in the yellow circle corresponds to genes exclusive for miR-3666. The intersection area of the two circles represents the number of target genes shared by both FOXP2 and miR-3666. Enriched heatmap of:

B. Gene Ontology Biological process (GOBP) terms.

C. KEGG pathways and

D. Enriched neurological disorders schizophrenia and autism. Multiple test corrected p-values are represented in color coded heatmaps. The higher the color intensity towards red, the greater is the significance, while higher color intensity towards yellow represents lower significance. Gray represents insignificant p-value. 
Supplementary Table $\mathbf{S} 4$ List of selected 30 genes full name and symbols

\begin{tabular}{|c|c|}
\hline Gene name & Gene symbol \\
\hline $\begin{array}{l}\text { Achaete-Scute Family BHLH Transcription } \\
\text { Factor I }\end{array}$ & ASCLI \\
\hline Brain-Derived Neurotrophic Factor & BDNF \\
\hline Cyclin D3 & CCND3 \\
\hline Cadherin 2 & $\mathrm{CDH} 2$ \\
\hline Contactin Associated Protein-Like 2 & CNTNAP2 \\
\hline CAMP Responsive Element Binding Protein I & CREBI \\
\hline DiGeorge Syndrome Critical Region Gene 2 & DGCR2 \\
\hline Disrupted In Schizophrenia I & DISCI \\
\hline Ephrin B2 & EFNB2 \\
\hline Empty Spiracles Homeobox 2 & $\mathrm{EMX} 2$ \\
\hline Erb-B2 Receptor Tyrosine Kinase 4 & ERBB4 \\
\hline Forkhead Box PI & FOXPI \\
\hline Hes Family BHLH Transcription Factor I & HESI \\
\hline Insulin Like Growth Factor I & IGFI \\
\hline LIM Domain Only 4 & LMO4 \\
\hline $\begin{array}{l}\text { MET Proto-Oncogene, Receptor Tyrosine } \\
\text { Kinase }\end{array}$ & MET \\
\hline NudE Neurodevelopment Protein I Like I & NDELI \\
\hline Neuronal Differentiation I & NEURODI \\
\hline Neurogenin I & NGNI \\
\hline Neurogenin 2 & NGN2 \\
\hline Neuregulin I & NRGI \\
\hline Paired Box 6 & PAX6 \\
\hline POU Class 3 Homeobox 2 & POU3F2 \\
\hline $\begin{array}{l}\text { Protein Tyrosine Phosphatase, Non-Receptor } \\
\text { Type II }\end{array}$ & PTPNII \\
\hline Protein Tyrosine Phosphatase, Receptor Type J & PTPRJ \\
\hline Synaptosome Associated Protein $25 \mathrm{kDa}$ & SNAP25 \\
\hline SRY (Sex Determining Region Y)-Box 2 & sox2 \\
\hline SRY (Sex Determining Region Y)-Box 2I & sox21 \\
\hline SRY (Sex Determining Region Y)-Box 4 & sox4 \\
\hline Transcription Factor 4 & TCF4 \\
\hline
\end{tabular}

SupplementaryTable S5 List of selected genes linked to neurodevelopmental disorders schizophrenia and/or autism

\begin{tabular}{ll}
\hline Schizophrenia candidate genes & Autism candidate genes \\
\hline BDNF & BDNF \\
DGCR2 & CNTNAP2 \\
ERBB4 & ERBB4 \\
FOXP2 & FOXPI \\
NRGI & FOXP2 \\
& MET \\
& PAX6 \\
& PTPNII \\
& SNAP25 \\
& TCF4 \\
\hline
\end{tabular}
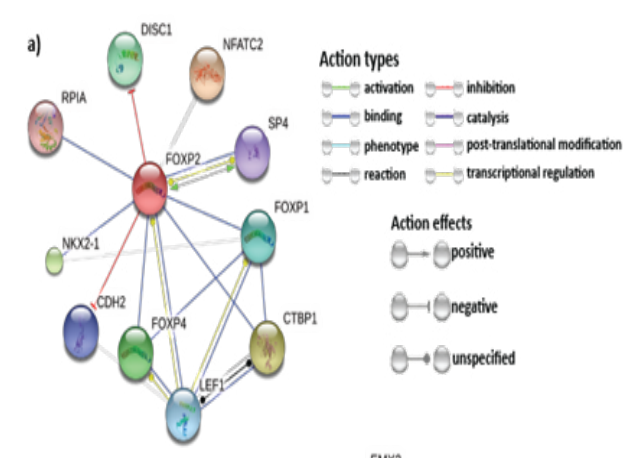

$\Rightarrow$ reaction $=-$ transcriptional tepoutation

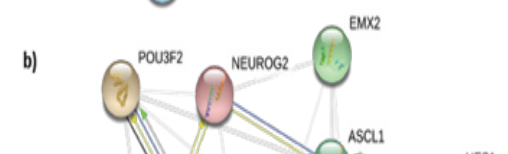

Action effects

$\Theta$ Opositive

O- Onegative

O- Ounspecified

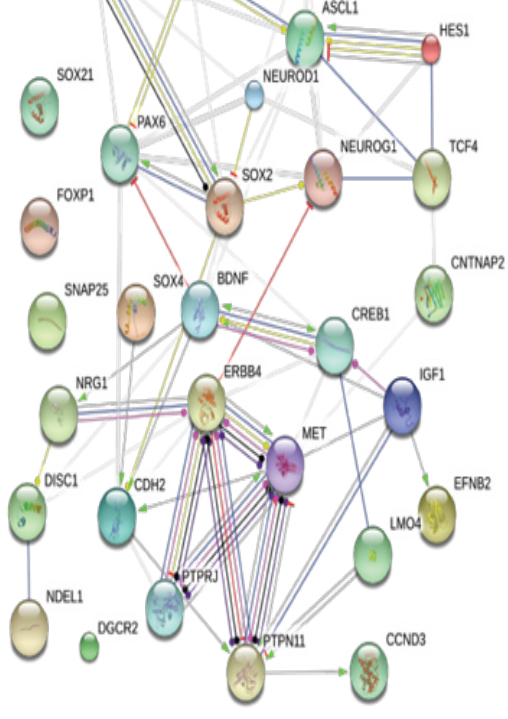

Figure 3 STRING network displaying
a. FOXP2 interactions and
b. Interactions among 30 selected genes.

The network nodes represent proteins whereas the edges represent proteinprotein associations. Small nodes represent protein of unknown 3D structure whereas large nodes mean some 3D structure is known or predicted. The colored nodes are for query proteins and first shell of interactors whereas white nodes are second shell of interactors. 


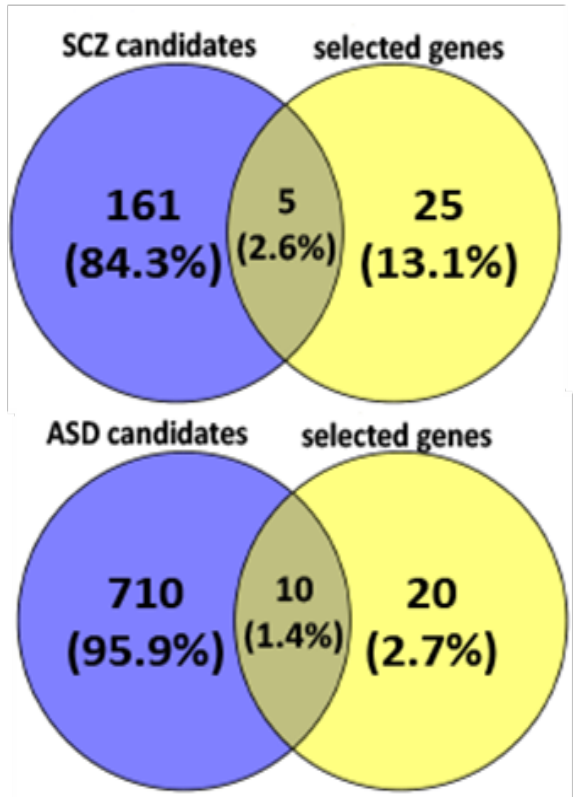

Figure 4 Venn diagrams representation of common genes between

a. Schizophrenia candidate genes and our list of selected genes and

b. Autism candidate genes and our list of selected genes.

The left circle represents disease candidate genes, where the number in the blue circle corresponds to genes exclusive for the disease. The right circle represents our selected genes, where the number in the yellow circle corresponds to genes exclusive for the selected genes. The intersection area of the two circles represents the number of selected genes that are also disease candidate genes.

MiR-3666 directly or indirectly regulates FOXP2 functions in neurite outgrowth and cortical patterning

As model 1 (Figure 5A) proposes, miR-3666 regulates neurite outgrowth, axon guidance and synaptic plasticity by regulating the gene EFNB2, a well-validated ChIP-Seq target of FOXP2. miR3666 can regulate proper cortical patterning through regulation of the common target gene $E M X 2$ (empty spiracles homolog 2) and miR3666 target PAX6 (paired box 6). The initial regional pattern of the neocortex is established by the distinct spatial distribution of PAX6 and EMX2. ${ }^{69}$ Additionally, model 2 (Figure 5B) demonstrates miR3666 may enhance FOXP2 suppression of LMO4 (LIM domain-only 4) by inhibiting the common target gene, CREBI (cAMP responsive element binding protein 1). LMO4 gene shows asymmetric expression in the embryonic human brain possibly due to repression by FOXP2 and hence plays important roles in cortical patterning. ${ }^{68} \mathrm{LMO} 4$ is known to form a complex with CREB. ${ }^{80}$

Analysis of GSE28633 ${ }^{23}$ revealed the expression changes of genes of models 1 and $2 . \log _{2}$-transformed and median centered expression values were visualized as a heatmap using a color coded scale. FOXP2 shows relatively higher expression in neuroectodermal stage (NE) and in differentiated neurons (DN) and lower expression in human embryonic stem cells (hESCs). As expected, during differentiation, we see the upregulation of genes EFNB2, PAX6 and EMX2 in NE and SOX4 in DN (Figure 6). Besides regulating FOXP2 functions by binding to its targets, model 1 (Figure 5A) shows miR-3666 may also regulate the expression of FOXP2 itself. POU3F2 (POU class 3 homeobox 2, aka Brn-2) has been known to bind and activate FOXP $2{ }^{81}$ Besides activating NEUROG2 $2{ }^{82,83}$ PAX6 can also induce the expression of POU3F $2{ }^{84}$ and FOXP $2{ }^{85}$ Hence, we infer that miR-
3666 can therefore regulate the expression of $F O X P 2$ by regulating its targets, the FOXP2 activators PAX6 and POU3F2.

\section{FOXP2 and miR-3666 may be responsible for the pa- thogenesis of schizophrenia}

Schizophrenia (SCZ) is a severe and chronic neuropsychiatric disorder; it is reported to have a lifetime prevalence of approximately $1 \% .{ }^{86}$ This neurodevelopmental disorder involves multiple genes that may be directly or indirectly modulated by FOXP 2 and miR3666. As model 3 (Figure 5C) shows, the candidate gene ERBB $4,^{87}$ being a common target gene, may be directly regulated by miR3666 and FOXP2. However, miR-3666 and FOXP2 can regulate the levels of candidate genes $B D N F$ (Brain-Derived Neurotrophic Factor), ${ }^{88}$ DGCR2 (DiGeorge syndrome critical region gene 2), ${ }^{89}$ $N R G 1^{60}$ through common target CREB1. This relation is evident from the GEO2R analysis of GSE17612 ${ }^{90}$ expression data where up regulation of $F O X P 2\left(\log _{2} \mathrm{FC}=0.54360366\right)$ and downregulation of BDNF $\left(\log _{2} \mathrm{FC}=-0.54217872\right)$ is observed in these cells (Figure 7). We assume the upregulation of FOXP2 may be responsible for low BDNF levels; FOXP2 and miR-3666 may indirectly repress BDNF by inhibition of CREB1. In the BDNF expression profile, one sample shows much higher expression level compared to the rest, which may be due to age-related differences or the use of anti-psychotic drugs. ${ }^{88,91,92}$ Moreover, FOXP2 probably directly inhibit DISC1 (Disrupted-In-Schizophrenia 1), ${ }^{93}$ whereas miR-3666 may indirectly inhibit it by repressing NDEL1 and hence disrupting DISC1-NDEL1 interaction. ${ }^{63}$

\section{FOXP2 and miR-3666 may be responsible for the pa- thogenesis of autism and ASD}

Autism and ASD are developmental disorders with three core symptoms: "deficits in social interactions and understanding; aberrant communication and/or language development; and restricted interests and repetitive, stereotyped behaviors ${ }^{94 "}$ Autism candidate genes $E R B B 4^{95}$ and $B D N F^{96,97}$ have been associated with ASD. As shown in model 3 (Figure 5C). ERBB4 may be directly co-regulated by miR3666 and FOXP2 since it is a common target; whereas BDNF levels may be indirectly regulated via common target CREB1. Therefore, these interactions have not been shown in model 4 (Figure 5D) again. FOXP1 (forkhead box protein P1) deletions ${ }^{98,99}$ and increase ${ }^{100}$ have both been associated with ASD. Both FOXP1 $1^{101}$ and FOXP2 can downregulate CNTNAP2 (Contactin-associated protein-like 2), ${ }^{100,102}$ another candidate gene of ASD. ${ }^{67}$ Chien et al. ${ }^{100}$ hypothesized that enhanced FOXP1 expression can increase the expression of FOXP2 through a feedback mechanism, which in-turn may then lead to the reduction of CNTNAP2 levels and result in ASD. Hence, interactions among FOXP1, FOXP2 and CNTNAP2 genes may be responsible for the pathogenesis of syndromic and non-syndromic ASD. ${ }^{100}$ As model 4 (Figure 5D) shows, miR-3666 can play an "enemy" role to FOXP2 by repressing FOXP1 and removing its inhibitory effect on CNTNAP2 or it may inhibit FOXP1 expression and affect the modulatory roles of FOXP2 that requires FOXP1-FOXP2 dimerization. FOXP2 and miR-3666 can also jointly affect the levels of common targets $M E T$ (MET receptor tyrosine kinase), TCF4, SNAP25 (synaptosomalassociated protein of $25 \mathrm{kDa}$ ) and $P T P N 11$, which are candidate genes for ASD. ${ }^{103-107}$ PAX6 regulation by miR-3666 is not only important to maintain the levels of FOXP2 but also to prevent the development of autism and related disorders, as PAX6 is also a candidate gene for ASD. ${ }^{108}$

Relations in above models are also supported by the transcriptomic expression data. GEO2R analysis of GSE38322 $2^{109,110}$ 
revealed the upregulation of TCF4 $\left(\log _{2} \mathrm{FC}=0.629\right)$ (Figure $\left.8 \mathrm{~A}\right)$ and downregulation of SNAP25 ( $\left.\log _{2} \mathrm{FC}=-0.828\right)$ (Figure $\left.8 \mathrm{~B}\right)$. GEO2R analysis of GSE29691 also revealed the up regulation of TCF4 $\left(\log _{2} \mathrm{FC}=0.55079923\right)$ (Figure $\left.8 \mathrm{C}\right)$ and down regulation of PTPN11 $\left(\log _{2} \mathrm{FC}=-0.53875346\right)$ (Figure 8D). Even though low levels of TCF4 has resulted in Pitt-Hopkins Syndrome (PHS) ${ }^{104,111}$ a disease related to autism with common symptoms, high levels of TCF4 has been observed in patients afflicted with SCZ. ${ }^{112,113}$ Since SCZ and autism are closely related, it may be deduced that high levels of TCF4 may lead to development of autism or related disorders. GEO2R analysis of GSE6575 $5^{114}$ did not show any significant differential expression of our selected genes, however, driver gene analysis of GSE6575 (Figure 9) revealed significant $(\mathrm{p}<0.05)$ down regulation of CNTNAP2 and TCF4 but no upregulated genes. These observations are in concordance to our models' suggestion of the levels of candidate genes associated with the disorders. It is not surprising that the expression data used as evidence for autism candidate gene levels did not show differential expression of FOXP2 levels in autism patients. This may be due to the use of blood samples in the experiment related to GSE29691, as FOXP2 is not significantly expressed in blood. ${ }^{115}$ Also, since postmortem brains are used, the expression may be too low for detection. A previous study attempted to measure the mRNA level of FOXP2 in lymphoblastoid cell lines using RT-qPCR, but the mRNA levels were too low to be detected. ${ }^{100}$

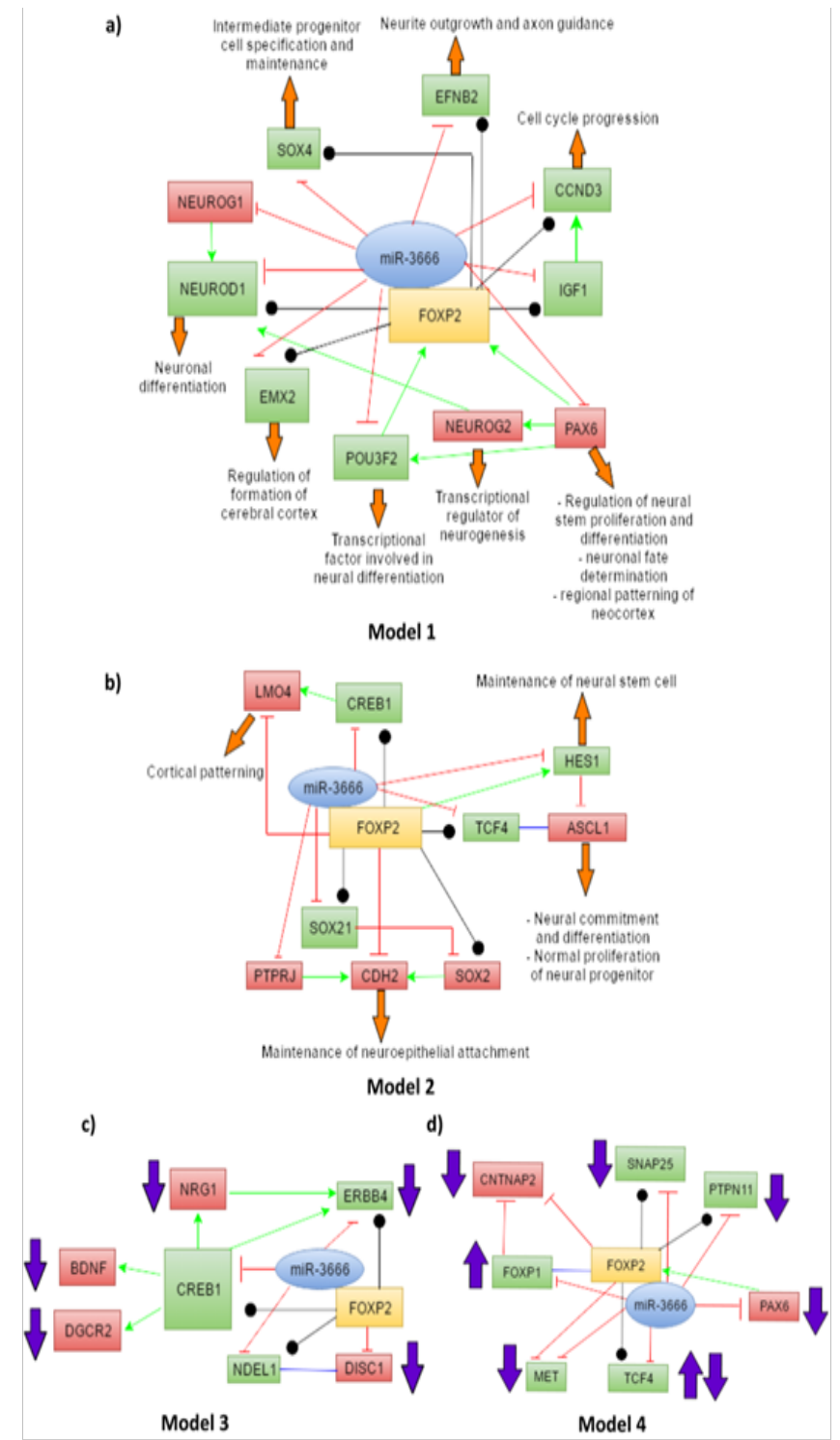

Figure 5 Models depicting the possible role of miR-3666 in modulation of FOXP2 functions in neurodevelopment in pathogenesis of neurological disorders.

A. Model I depicts direct regulation by miR-3666 of FOXP2 and common targets.

B. Model 2 depicts indirect regulation by miR-3666 of FOXP2 functions.

C. Model 3 represents co-regulation of miR-3666 and FOXP2 in pathogenesis of schizophrenia and

D. Model 4 represents co-regulation of miR-3666 and FOXP2 in pathogenesis of autism and related disorders. miR-3666 is shown in blue whereas FOXP2 is shown in yellow.

The common target genes are shown in green whereas targets that are not shared by miR-3666 and FOXP2 are shown in red. The green arrows represent directional activation and the blunt-ended red lines show directional inhibition. The black lines with circled ends represent interaction and the blue lines represent binding (or dimerization). The orange block arrows associated with the genes show the function of the particular genes and the purple block arrows show levels of candidate genes found in diseased individuals. 


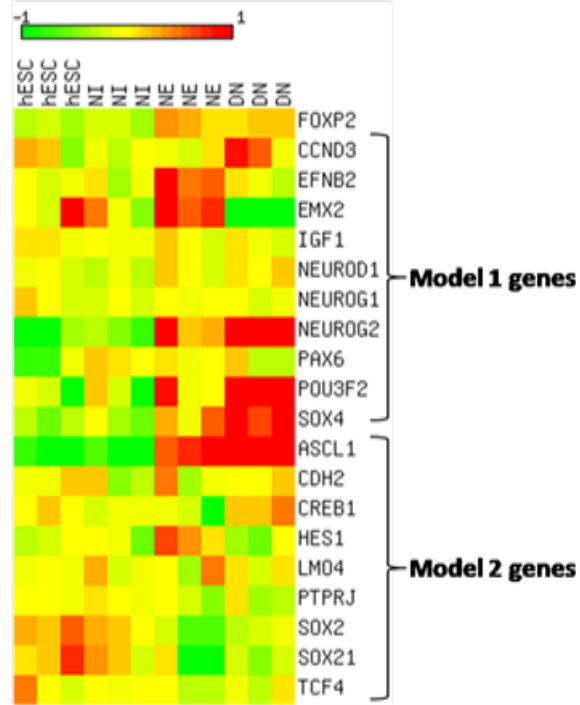

Figure 6 Heatmap visualization of the expression profile of model I and 2 genes during neural differentiation.

Log2 expression values of each gene is subtracted from row median expression and represented in color coded heatmap. The rows represent genes whereas the columns represent samples corresponding to:

I. hESC: human embryonic stem cell

II. NI: neural induction

III. NE: neural ectoderm and

IV. DN: differentiated neurons

A color coded scale from 2 to 12 represents expression values where color intensity towards red corresponds to higher expression from median and color intensity towards green corresponds to lower expression from median expression value and yellow corresponds to median expression.

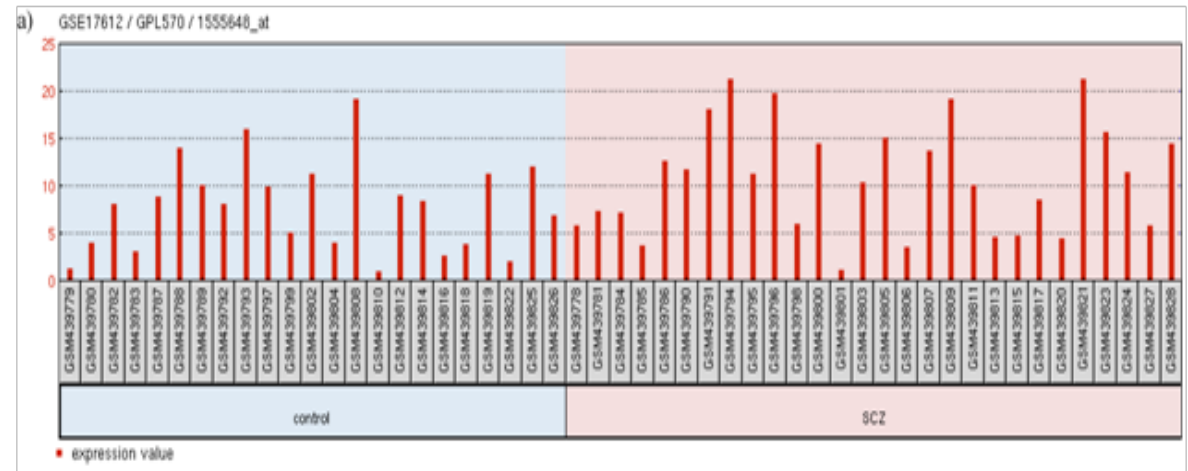

b) GSE17612/ GPL.570/ 239967_zt

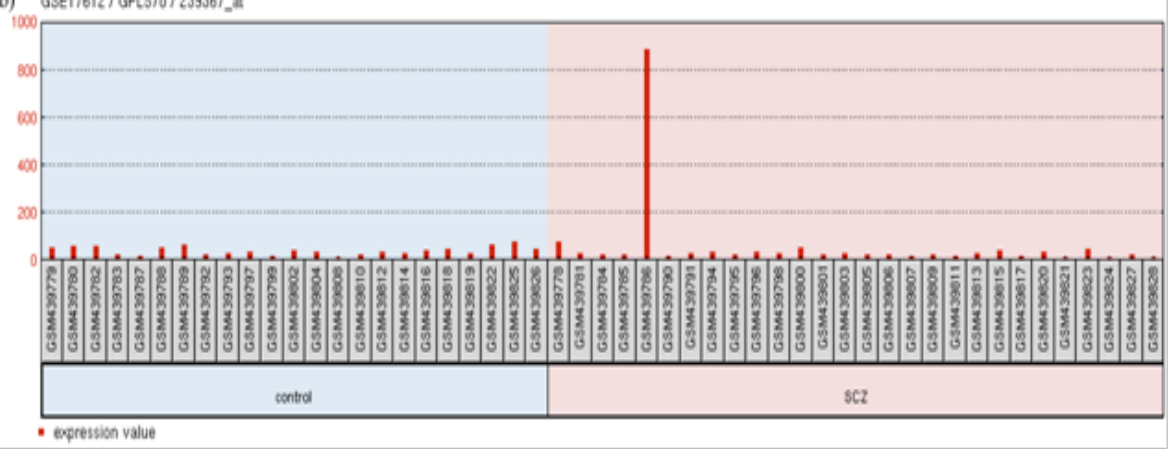

Figure 7 Expression profile of

A. FOXP2 and

B. BDNF in GSEI76I2.

The title shows GEO accession number (GSEI76I2), platform ID (GPL570) and the probe ID (I555648_at for FOXP2 and 239367_at for BDNF). The groups shown are (I) control and (2) SCZ (shizophrenia). Each group contains particular samples as designated by their GSM IDs. The red bars represent the expression measurement extracted from the MAS5.0 signal intensity values of the samples. 

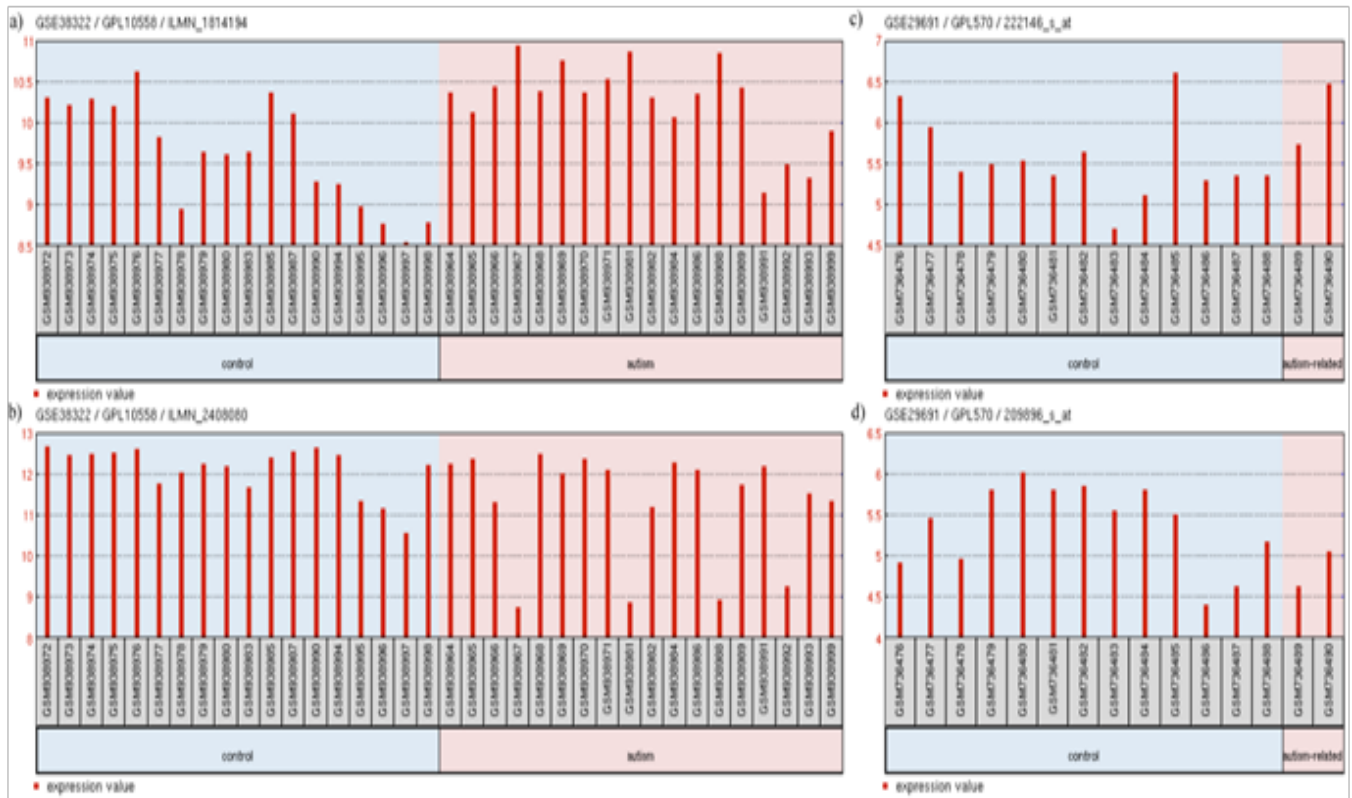

Figure 8 Expression profile of
A. TCF4
B. SNAP25 in GSE38322
C. TCF4 and
D. PTPNII in GSE2969I.

The profile diagram is titled in the fomat "GEO accession number/platform ID/probe ID". The groups shown are (I) control and (2) autism-related. Each group contains particular samples as designated by their GSM IDs. The red bars in GSE38322 represent the expression measurement extracted from the quantile normalized, variance stabilized, signal intensity values of the samples whereas the red bars in GSE2969I represent the expression measurement extracted from the Log2 GCRMA signal intensity values of the samples.

\section{FAM132B \\ IDO1 \\ $\operatorname{TSTA3}$ \\ CHTHAP 2 \\ $\mathrm{ABCC13}$ \\ HEMGI \\ CXCL10 \\ TCF4 \\ RSAD2 \\ PRSS 33}

\section{p-value for enrichment}

0.0

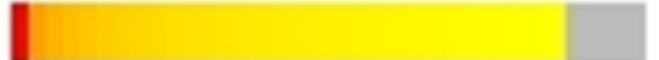 \\ Significant \\ non-significant}

Figure 9 Heatmap of selected down regulated driver genes.

Driver gene analysis from GEO expression dataset GSE6575.P-value of significance as candidate driver gene is represented in a color coded scale in the heatmap. Color towards red indicates more significantly down regulated driver genes, where as color towards yellow indicates less significantly down regulated genes and grey indicates non-significant genes. Our selected genes are highlighted in square box.

\section{Conclusion}

This study demonstrates that intronic miRNA, miR-3666 and its host gene, FOXP2, coincides with a functional relation in neurodevelopment, as deduced from literature mining, expression data analysis and functional enrichment analysis of the common target genes. Further literature mining and interaction studies show how miR-3666 may regulate FOXP2 functions as an "enemy" or "partner"; based on which four models were developed. Neurodevelopment being a complex biological process requires precise regulation; these models suggest mechanisms in which miR-3666 can modulate $F O X P 2$ functions by directly or indirectly affecting the expression of FOXP2 target genes to ensure the precise spatial and temporal regulation of genes associated with neurodevelopment. The models also show how miR-3666 and FOXP2 may be associated with the neurodevelopmental disorders schizophrenia and autism. Microarray expression data were analyzed which support some interactions between FOXP2 and its targets as portrayed in the models. Further validation of these models by in vitro and in vivo experiments would help in the development of more effective stem cell therapies, which is especially attractive due to limited regenerative capacity of neurons in mammals. ${ }^{116}$ Besides stem-cell therapies, an understanding of the function of miR-3666 may be useful for the designing of miRNAbased therapeutics. For example, in our study we find that miR-3666 and FOXP2 inhibit MET expression. Since reduced MET levels in the brain have been associated with autism, anti-miR-3666 may be a potential drug to offset the inhibitory effects of FOXP2 and return MET to normal levels. Additionally, the results may be implicated in development of therapeutics against neurodevelopmental disorders. 


\section{Acknowledgements}

We acknowledge the research grants from University Grants Commission (to Dhaka University). SMM was recipient of National Science and Technology Fellowship (grant ID:73) from Ministry of Science and Technology, Government of Bangladesh.

\section{Conflict of interest}

The author declares no conflict of interest.

\section{References}

1. Smith B, Treadwell J, Zhang D, et al. Large-scale expression analysis reveals distinct microRNA profiles at different stages of human neurodevelopment. PLoS One. 2010;5(6):e11109.

2. Sioud M. RNA therapeutics: function, design, and delivery. Preface. $M e-$ thods Mol Biol. 2010;629:5-7.

3. Gao X, Qiao Y, Han D, et al. Enemy or partner: Relationship between intronic micrornas and their host genes. IUBMB Life. 2012;64(10):835840 .

4. Gray KA, Yates B, Seal RL, et al. Genenames.org: the HGNC resources in 2015. Nucleic Acids Res. 2015;43(Database issue):D1079-D1085.

5. Fishilevich S, Zimmerman S, Kohn A, et al. Genic insights from integrated human proteomics in Gene cards. Database (Oxford). 2016;2016:baw030.

6. Consortium UP. UniProt: a hub for protein information. Nucleic Acids Res. 2014;43(Database issue):D204-D212

7. Spiteri E, Konopka G, Coppola G, et al. Identification of the transcriptional targets of $F O X P 2$, a gene linked to speech and language, in developing human brain. Am J Hum Genet. 2007;81(6):1144-1157.

8. Vernes SC, Spiteri E, Nicod J, et al. High-throughput analysis of promoter occupancy reveals direct neural targets of FOXP2, a gene mutated in speech and language disorders. Am J Hum Genet. 2007;81(6):1232-1250.

9. Wang G, Cai C, Chen L. MicroRNA-3666 regulates thyroid carcinoma cell proliferation via MET. Cell Physiol Biochem. 2016;38(3):1030-1039.

10. Li L, Han LY, Yu M, et al. Pituitary tumor-transforming gene 1 enhances metastases of cervical cancer cells through miR-3666-regulated ZEB1. Tumour Biol. 2015; [Epub ahead of print]

11. Truscott M, Islam AB, Lightfoot $\mathrm{J}$, et al. An intronic microRNA links $\mathrm{Rb}$ E2F and EGFR signaling. PLoS Genet. 2014;10(7):e1004493.

12. Truscott M, Islam AB, López-Bigas N, et al. Mir-11 limits the proapoptotic function of its host gene, dE2f1. Genes Dev. 2011;25(17):1820-1834

13. Rooij EV, Olson EN. MicroRNA therapeutics for cardiovascular disease: opportunities and obstacles. Nat Rev Drug Discov. 2012;11(11):860-872.

14. Wang J, Chen J, Sen S. MicroRNA as biomarkers and diagnostics. J Cell Physiol. 2016;231(1):25-30.

15. Lewis BP, Burge CB, Bartel DP. Conserved seed pairing, often flanked by adenosines, indicates that thousands of human genes are microRNA targets. Cell. 2005;120(1):15-20.

16. Vlachos IS, Paraskevopoulou MD, Karagkouni D, et al. DIANA-TarBase v7.0: indexing more than half a million experimentally supported miRNA:mRNA interactions. Nucleic Acids Res. 2015;43(Database issue):D153-D159.

17. Krek A, Grün D, Poy MN, et al. Combinatorial microRNA target predictions. Nat Genet. 2005;37(5):495-500.
18. Xiao F, Zuo Z, Cai G, et al. miRecords: an integrated resource for microRNA-target interactions. Nucleic Acids Res. 2009;37(Database issue) D105-D110.

19. Rosenbloom KR, Sloan CA, Malladi VS, et al. ENCODE data in the UCSC Genome Browser: year 5 update. Nucleic Acids Res. 2013;41(Database issue):D56-63.

20. Quinlan AR, Hall IM. BEDTools: a flexible suite of utilities for comparing genomic features. Bioinformatics. 2010;26(6):841-842.

21. Flicek P, Ahmed I, Amode MR, et al. Ensembl 2013. Nucleic Acids Res. 2013;41(Database issue):D48-D55.

22. Oliveros JC. VENNY. An interactive tool for comparing lists with Venn Diagrams; 2007.

23. Fathi A, Hatami M, Hajihosseini V, et al. Comprehensive gene expression analysis of human embryonic stem cells during differentiation into neural cells. PLoS One. 2011;6(7):e22856.

24. Pavlidis P, Noble WS. Matrix2png: a utility for visualizing matrix data Bioinformatics. 2003;19(2):295-296.

25. Llamas CP, Bigas NL. Gitools: analysis and visualisation of genomic data using interactive heat-maps. PLoS One. 2011;6(5):e19541.

26. Edgar R, Domrachev M, Lash AE. Gene Expression Omnibus: NCB gene expression and hybridization array data repository. Nucleic Acids Res. 2002;30(1):207-210.

27. Barrett T, Wilhite SE, Ledoux P, et al. NCBI GEO: archive for functional genomics data sets--update. Nucleic Acids Res. 2013;41(Database issue):D991-995.

28. Ashburner M, Ball CA, Blake JA, et al. Gene ontology: tool for the unification of biology. The gene ontology consortium. Nat Genet. $2000 ; 25(1): 25-29$

29. Hubbard TJ, Aken BL, Beal K, et al. Ensembl 2007. Nucleic Acids Res 2007;35(Database issue):D610-D617.

30. Kanehisa M, Araki M, Goto S, et al. KEGG for linking genomes to life and the environment. Nucleic Acids Res. 2008;36(Database issue):D480D484.

31. Benjamini Y, Drai D, Elmer G, et al. Controlling the false discovery rate in behavior genetics research. Behav Brain Res. 2001;125(1-2):279-284.

32. Benjamini Y, Hochberg Y. Controlling the false discovery rate: a practical and powerful approach to multiple testing. Journal of the royal statistical society. Series B (Methodological). 1995;57(1):289-300.

33. Jia P, Sun J, Guo AY, et al. SZGR: a comprehensive schizophrenia gene resource. Mol Psychiatry. 2010;15(5):453-462.

34. Xu LM, Li JR, Huang Y, et al. AutismKB: an evidence-based knowledgebase of autism genetics. Nucleic Acids Res. 2012;40(Database issue):D1016-D1022.

35. Basu SN, Kollu R, Basu SB. AutDB: a gene reference resource for autism research. Nucleic acids research. Nucleic Acids Res. 2009;37(Database issue):D832-D836

36. Jensen LJ, Kuhn M, Stark M, et al. STRING 8-a global view on proteins and their functional interactions in 630 organisms. Nucleic Acids Res. 2009;37(Database issue):D412-D416.

37. Szklarczyk D, Franceschini A, Wyder S, et al. STRING v10: protein-protein interaction networks, integrated over the tree of life. Nucleic Acids Res. 2015;43(Database issue):D447-D452.

38. Stark C, Breitkreutz BJ, Reguly T, et al. BioGRID: a general repository for interaction datasets. Nucleic Acids Res. 2006;34(Database issue):D535-D539. 
39. Orchard S, Ammari M, Aranda B, et al. The MIntAct project--IntAct as a common curation platform for 11 molecular interaction databases. $\mathrm{Nu}$ cleic Acids Res. 2014;42(Database issue):D358-D363.

40. Khadem FV, Gadian DG, Copp A, et al. FOXP2 and the neuroanatomy of speech and language. Nat Rev Neurosci. 2005;6(2):131-138.

41. Marcus GF, Fisher SE. FOXP2 in focus: What can genes tell us about speech and language? Trends Cogn Sci. 2003;7(6):257-262.

42. Takahashi H, Takahashi K, Liu FC. FOXP Genes, neural development, speech and language disorders. Adv Exp Med Biol. 2009;665:117-129.

43. Shu W, Cho JY, Jiang Y, et al. Altered ultrasonic vocalization in mice with a disruption in the Foxp2 gene. Proc Natl Acad Sci U S A. 2005;102(27):9643-9648.

44. French CA, Groszer M, Preece C, et al. Generation of mice with a conditional Foxp2 null allele. Genesis. 2007;45(7):440-446.

45. Kurt S, Fisher SE, Ehret G. Foxp 2 mutations impair auditory-motor association learning. PLoS One. 2012;7(3):e33130.

46. Lai CS, Fisher SE, Hurst JA, et al. A forkhead-domain gene is mutated in a severe speech and language disorder. Nature. 2001;413(6855):519-523.

47. Watkins KE, Dronkers NF, Vargha-Khadem F. Behavioural analysis of an inherited speech and language disorder: comparison with acquired aphasia. Brain. 2002;125(Pt 3):452-464.

48. Vernes SC, Nicod J, Elahi FM, et al. Functional genetic analysis of mutations implicated in a human speech and language disorder. Hum Mol Genet. 2006;15(21):3154-3167.

49. Li T, Zeng Z, Zhao Q, et al. FOXP2 is significantly associated with schizophrenia and major depression in the Chinese Han population. World $J$ Biol Psychiatry. 2013;14(2):146-150.

50. Tolosa A, Sanjuán J, Dagnall AM, et al. FOXP2 gene and language impairment in schizophrenia: association and epigenetic studies. BMC Med Genet. 2010;11:114.

51. Španiel F, Horáček J, Tintěra J, et al. Genetic variation in FOXP2 alters grey matter concentrations in schizophrenia patients. Neurosci Lett. 2011;493(3):131-135

52. Sanjuán J, Tolosa A, González JC, et al. Association between FOXP2 polymorphisms and schizophrenia with auditory hallucinations. Psychiatr Genet. 2006;16(2):67-72.

53. Casey JP, Magalhaes T, Conroy JM, et al. A novel approach of homozygous haplotype sharing identifies candidate genes in autism spectrum disorder. Hum Genet. 2012;131(4):565-579.

54. Schaaf CP, Sabo A, Sakai Y, et al. Oligogenic heterozygosity in individuals with high-functioning autism spectrum disorders. Hum Mol Genet. 2011;20(17):3366-3375.

55. Li H, Yamagata T, Mori M, et al. Absence of causative mutations and presence of autism-related allele in FOXP2 in Japanese autistic patients. Brain Dev. 2005;27(3):207-210.

56. Gong X, Jia M, Ruan Y, et al. Association between the FOXP2 gene and autistic disorder in Chinese population. Am J Med Genet B Neuropsychiatr Genet. 2004;127B(1):113-116.

57. Konopka G, Friedrich T, Turak JD, et al. Human-specific transcriptional networks in the brain. Neuron. 2012;75(4):601-617.

58. Konopka G, Bomar JM, Winden K, et al. Human-specific transcriptional regulation of CNS development genes by FOXP2. Nature. 2009;462(7270):213-217.

59. Ohnuma S, Harris WA. Neurogenesis and the cell cycle. Neuron. 2003;40(2):199-208
60. Banerjee A, Macdonald ML, Winter KEB, et al. Neuregulin 1-erbB4 pathway in schizophrenia: From genes to an interactome. Brain Res Bull. 2010;83(3-4):132-139.

61. Binder DK, Scharfman HE. Brain-derived neurotrophic factor. Growth Factors. 2004;22(3):123-131

62. Castro DS, Martynoga B, Parras C, et al. A novel function of the proneural factor Ascl1 in progenitor proliferation identified by genome-wide characterization of its targets. Genes Dev. 2011;25(9):930-945.

63. Kamiya A, Tomoda T, Chang J, et al. DISC1- NDEL1/NUDEL protein interaction, an essential component for neurite outgrowth, is modulated by genetic variations of DISC1. Hum Mol Genet. 2006;15(22):3313-3323.

64. Mo Z, Zecevic N. Is Pax6 critical for neurogenesis in the human fetal brain? Cereb Cortex. 2008;18(6):1455-1465.

65. Ohtsuka T, Shimojo H, Matsunaga M, et al. Gene expression profiling of neural stem cells and identification of regulators of neural differentiation during cortical development. Stem Cells. 2011;29(11):1817-1828.

66. Pacary E, Martynoga B, Guillemot F. Crucial first steps: the transcriptional control of neuron delamination. Neuron. 2012;74(2):209-211.

67. Peñagarikano O, Abrahams BS, Herman EI, et al. Absence of CNTNAP2 leads to epilepsy, neuronal migration abnormalities and core autism-related deficits. Cell. 2011;147(1):235-246.

68. Vernes SC, Oliver PL, Spiteri E, et al. FOXP2 regulates gene networks implicated in neurite outgrowth in the developing brain. PLoS Genet. 2011;7(7):e1002145.

69. Zhang J, Jiao J. Molecular biomarkers for embryonic and adult neural stem cell and neurogenesis. Biomed Res Int. 2015;2015:727542.

70. Chen C, Lee GA, Pourmorady A, et al. Orchestration of neuronal differentiation and progenitor pool expansion in the developing cortex by soxc genes. J Neurosci. 2015;35(29):10629-10642.

71. Forrest MP, Waite AJ, Martin-Rendon E, et al. Knockdown of human TCF4 affects multiple signaling pathways involved in cell survival, epithelial to mesenchymal transition and neuronal differentiation. PLoS One. 2013;8(8):e73169.

72. Chen H, Thiagalingam A, Chopra H, et al. Conservation of the Drosophila lateral inhibition pathway in human lung cancer: a hairy-related protein (HES-1) directly represses achaete-scute homolog-1 expression. Proc Natl Acad Sci U S A. 1997;94(10):5355-5360.

73. Somasundaram K, Reddy SP, Vinnakota K, et al. Upregulation of ASCL1 and inhibition of Notch signaling pathway characterize progressive astrocytoma. Oncogene. 2005;24(47):7073-7083.

74. Devanna P, Middelbeek J, Vernes SC. FOXP2 drives neuronal differentiation by interacting with retinoic acid signaling pathways. Front Cell Neurosci. 2014;8:305.

75. Holmberg J, Hansson E, Malewicz M, et al. SoxB1 transcription factors and Notch signaling use distinct mechanisms to regulate proneural gene function and neural progenitor differentiation. Development. 2008;135(10):1843-1851

76. Kamachi Y, Kondoh H. Sox proteins: regulators of cell fate specification and differentiation. Development. 2013;140(20):4129-4144.

77. Rousso DL, Pearson CA, Gaber ZB, et al. Foxp-mediated suppression of $\mathrm{N}$-cadherin regulates neuroepithelial character and progenitor maintenance in the CNS. Neuron. 2012;74(2):314-330.

78. Balsamo J, Leung T, Ernst H, et al. Regulated binding of PTP1B-like phosphatase to N-cadherin: control of cadherin-mediated adhesion by dephosphorylation of beta-catenin. J Cell Biol. 1996;134(3):801-813. 
79. Rhee J, Lilien J, Balsamo J. Essential tyrosine residues for interaction of the non-receptor protein-tyrosine phosphatase PTP1B with $\mathrm{N}$-cadherin. $J$ Biol Chem. 2001;276(9):6640-6644.

80. Kashani AH, Qiu Z, Jurata L, et al. Calcium Activation of the LMO4 Transcription Complex and Its Role in the Patterning of Thalamocortical Connections. J Neurosci. 2006;26(32):8398-8408.

81. Maricic T, Günther V, Georgiev O, et al. A recent evolutionary change affects a regulatory element in the human FOXP2 gene. Mol Biol Evol. 2013;30(4):844-852.

82. Scardigli R, Schuurmans C, Gradwohl G, et al. Crossregulation between Neurogenin 2 and pathways specifying neuronal identity in the spinal cord. Neuron. 2001;31(2):203-217.

83. Scardigli R, Bäumer N, Gruss P, et al. Direct and concentration-dependent regulation of the proneural gene Neurogenin2 by Pax6. Development. 2003;130(14):3269-3281.

84. Ninkovic J, Mezzadri AS, Jawerka M, et al. The BAF complex interacts with Pax6 in adult neural progenitors to establish a neurogenic cross-regulatory transcriptional network. Cell Stem Cell. 2013;13(4):403-418.

85. Coutinho P, Pavlou S, Bhatia S, et al. Discovery and assessment of conserved Pax6 target genes and enhancers. Genome Res. 2011;21(8):13491359.

86. Smyth AM, Lawrie SM. The neuroimmunology of schizophrenia. Clin Psychopharmacol Neurosci. 2013;11(3):107-117.

87. Silberberg G, Darvasi A, Kramarski RP, et al. The involvement of ErbB4 with schizophrenia: Association and expression studies. Am J Med Genet B Neuropsychiatr Genet. 2006;141B(2):142-148.

88. Nieto R, Kukuljan M, Silva H. BDNF and schizophrenia: from neurodevelopment to neuronal plasticity, learning, and memory. Front Psychiatry. 2013;4:45.

89. Shifman S, Levit A, Chen ML, et al. A complete genetic association scan of the 22q11 deletion region and functional evidence reveal an association between DGCR2 and schizophrenia. Hum Genet. 2006;120(2):160-170.

90. Maycox PR, Kelly F, Taylor A, et al. Analysis of gene expression in two large schizophrenia cohorts identifies multiple changes associated with nerve terminal function. Mol Psychiatry. 2009;14(12):1083-1094.

91. Muglia P, Vicente AM, Verga M, et al. Association between the BDNF gene and schizophrenia. Mol Psychiatry. 2003;8(2):146-147.

92. Green MJ, Matheson SL, Shepherd A, et al. Brain-derived neurotrophic factor levels in schizophrenia: a systematic review with meta-analysis. Mol Psychiatry. 2011;16(9):960-972.

93. Walker RM, Hill AE, Newman AC, et al. The DISC1 promoter: Characterization and regulation by FOXP2. Hum Mol Genet. 2012;21(13):28622872 .

94. Hu VW, Frank BC, Heine S, et al. Gene expression profiling of lymphoblastoid cell lines from monozygotic twins discordant in severity of autism reveals differential regulation of neurologically relevant genes. BMC Genomics. 2006;7:118.

95. Kaphzan H, Hernandez P, Jung JI, et al. Reversal of impaired hippocampal long-term potentiation and contextual fear memory deficits in Angelman syndrome model mice by ErbB inhibitors. Biol Psychiatry. 2012;72(3):182-190.

96. Bryn V, Halvorsen B, Ueland T, et al. Brain derived neurotrophic factor (BDNF) and autism spectrum disorders (ASD) in childhood. Eur J Paediatr Neurol. 2015;19(4):411-414.

97. Zheng Z, Zhang L, Zhu T, et al. Peripheral brain-derived neurotrophic factor in autism spectrum disorder: a systematic review and meta-analysis. Sci Rep. 2016;6:31241
98. Araujo DJ, Anderson AG, Berto S, et al. FoxP1 orchestration of ASD-relevant signaling pathways in the striatum. Genes Dev. 2015;29(20):20812096

99. Bacon C, Schneider M, Le Magueresse C, et al. Brain-specific Foxp1 deletion impairs neuronal development and causes autistic-like behaviour. Mol Psychiatry. 2015;20(5):632-639.

100. Chien WH, Gau SS, Chen CH, et al. Increased gene expression of FOXP in patients with autism spectrum disorders. Mol Autism. 2013;4(1):23.

101. Cuadrado PR, Ho J, Vernes SC. Shining a light on CNTNAP2: complex functions to complex disorders. Eur J Hum Genet. 2014;22(2):171-178.

102. Vernes SC, Newbury DF, Abrahams BS, et al. A functional genetic link between distinct developmental language disorders. $N$ Engl $\mathrm{J} \mathrm{Med}$. 2008;359(22):2337-2345.

103. Peng Y, Lu Z, Li G, et al. The autism-associated MET receptor tyrosine kinase engages early neuronal growth mechanism and controls glutamatergic circuits development in the forebrain. Mol Psychiatry. 2016;21(7):925-935.

104. Amiel J, Rio M, De Pontual L, et al. Mutations in TCF4, encoding a class I basic helix-loop-helix transcription factor, are responsible for $\mathrm{Pi}$ tt-Hopkins syndrome, a severe epileptic encephalopathy associated with autonomic dysfunction. Am J Hum Genet. 2007;80(5):988-993.

105. Blake DJ, Forrest M, Chapman RM, et al. TCF4, schizophrenia and pitt-hopkins syndrome. Schizophr Bull. 2010;36(3):443-447.

106. Braida D, Guerini FR, Ponzoni L, et al. Association between SNAP-25 gene polymorphisms and cognition in autism: functional consequences and potential therapeutic strategies. Transl Psychiatry. 2015;5:e500.

107. Pierpont EI, Pierpont ME, Mendelsohn NJ, et al. Genotype differences in cognitive functioning in Noonan syndrome. Genes Brain Behav. 2009;8(3):275-282.

108. Umeda T, Takashima N, Nakagawa R, et al. Evaluation of Pax6 mutant rat as a model for autism. PLoS One. 2010;5(12):e15500.

109. Ginsberg MR, Rubin RA, Falcone T, et al. Brain transcriptional and epigenetic associations with autism. PLoS One. 2012;7(9):e44736.

110. Ginsberg MR, Rubin RA, Natowicz MR. Patterning of regional gene expression in autism: new complexity. Sci Rep. 2013;3:1831.

111. Zweier C, Peippo MM, Hoyer J, et al. Haploinsufficiency of TCF4 causes syndromal mental retardation with intermittent hyperventilation (Pitt-Hopkins syndrome). Am J Hum Genet. 2007;80(5):994-1001.

112. Zhu X, Gu H, Liu Z, et al. Associations between TCF4 gene polymorphism and cognitive functions in schizophrenia patients and healthy controls. Neuropsychopharmacology. 2013;38(4):683-689.

113. Wirgenes KV, Sønderby IE, Haukvik UK, et al. TCF4 sequence variants and mRNA levels are associated with neurodevelopmental characteristics in psychotic disorders. Transl Psychiatry. 2012;2:e112.

114. Gregg JP, Lit L, Baron CA, et al. Gene expression changes in children with autism. Genomics. 2008;91(1):22-29.

115. Thomas AC, Frost JM, Ishida M, et al. The speech gene FOXP2 is not imprinted. J Med Genet. 2012;49(11):669-670.

116. Park KI, Himes BT, Stieg PE, et al. Neural stem cells may be uniquely suited for combined gene therapy and cell replacement: Evidence from engraftment of Neurotrophin-3-expressing stem cells in hypoxic-ischemic brain injury. Exp Neurol. 2006;199(1):179-190. 